\title{
Online Fault Diagnosis for Photovoltaic Modules Based on Probabilistic Neural Network
}

\author{
Ling Chen ${ }^{1 *}$, Wei Han², Yuhui Huang ${ }^{3}$, Xiang Cao ${ }^{1}$ \\ ${ }^{1}$ School of Physics and Electronic Electrical Engineering, Huaiyin Normal University, Huai' an 223300, China \\ ${ }^{2}$ State Grid Jiangsu Electric Power Co., Ltd. Huai'an Power Supply Branch, Huai’an 223001, China \\ ${ }^{3}$ Key Laboratory of Control of Power Transmission and Conversion, Ministry of Education, School of Electronic, Information \\ and Electrical Engineering, Shanghai Jiaotong University, Shanghai 200240, China
}

Corresponding Author Email: carolc11987@126.com

https://doi.org/10.18280/ejee.210309

Received: 8 March 2019

Accepted: 15 May 2019

\section{Keywords:}

photovoltaic $(P V)$ modules, fault diagnosis, probabilistic neural network $(P N N)$, backpropagation neural network $(B P N N)$

\begin{abstract}
Photovoltaic (PV) modules often suffer from various faults due to the harsh working environment. This paper proposes a PV fault diagnosis model based on probabilistic neural network (PNN), aiming to enhance the efficiency and reduce the maintenance cost of PV power stations. The influencing factors of PV faults were analyzed in details, and the output features of PV modules under fault states were simulated on MATLAB. Based on the simulation results, the fault types of PV modules were summed up, and a PNN-based PV fault diagnosis model was established. The effectiveness of our model was verified through simulation and experiment and compared with that of the diagnosis model based on backpropagation neural network (BPNN). The results show that our model can effectively detect four types of fault for PV modules, namely, short circuit, open circuit, abnormal degradation and partial shading, and enjoys high accuracy and robustness.
\end{abstract}

\section{INTRODUCTION}

In recent years, the photovoltaic (PV) technology has become increasingly popular as a green and clean power generation method [1]. Many measures have been taken to reduce the cost of PV power stations, namely, implementing maximum power point tracking (MPPT), and improving the conversion efficiency of PV modules and power inverter [2]. Apart from these measures, the PV power generation cost could be further reduced through effective identification of the faults in PV modules [3].

The existing fault diagnosis methods for PV modules fall into two categories: online diagnosis and offline diagnosis. Typical online diagnosis approaches include infrared imagery [4] and multi-sensor detection [5, 6]. The infrared imagery makes use of the apparent temperature differences between normal and fault PV modules. In this method, infrared images of PV modules are taken by infrared cameras, and used to judge the type and location of faults. During multi-sensor detection, voltage and current sensors are installed on one or several PV modules, and then the fault types and locations of these modules are analyzed based on the data collected by these sensors. These online diagnosis methods are more suitable for small PV power stations. If the PV power station is too large, quite a few infrared cameras and sensors need to be set up, pushing up the cost of PV power generation. Moreover, the cameras and sensors may operate abnormally under the harsh environment, which affects the accuracy of fault diagnosis.

The most representative offline diagnosis strategies are capacitance-to-ground (CTG) measurement [7] and time domain reflectometry (TDR) [8]. The former measures the CTG of the PV in series and thus locates the open circuit. The latter injects a pulse into the circuit of PV in series, analyzes the shape and delay of the return signal, and then identifies the type and location of faults. However, both CTG measurement and the TDR have a very limited scope of application, in that they must work offline and, on the site, require precision instruments to analyze waveforms, and only apply to PV modules in series rather than those in series-parallel connection.

The faults of PV modules are varied, unpredictable and heavily influenced by external factors, and thus difficult to be diagnosed [9-12]. Fortunately, the neural network (NN) can characterize the relations between the states and causes of PV faults with structures, connection weights and thresholds [13]. The faults of PV modules could be identified and classified accurately, after inputting the measured data to the trained $\mathrm{NN}$. One of the most suitable NNs for PV fault diagnosis is probabilistic neural network (PNN). This artificial neural network (ANN) is simple in structure and easy to train and implement [14]. The PNN-based fault diagnosis boasts a strong ability of nonlinear classification. During the diagnosis, the diagnosis spaces are mapped into fault modes, forming a diagnosis network with good fault-tolerance and an adaptive structure.

This paper proposes a PNN-based diagnosis model for PV modules, and collects suitable samples to train the PNN, aiming to improve the correctness and adaptability of the model. The effectiveness of our model was verified through both simulation and experiment.

The remainder of this paper is organized as follows: Section 2 sets up the inputs of PV fault diagnosis; Section 3 identifies the defects of backpropagation neural network (BPNN), and puts forward a PNN-based PV fault diagnosis model; Section 3 validates the proposed model through both simulation and experiment, and compares its diagnostic results with those of the BPNN; Section 4 wraps up this research with several 


\section{INPUTS OF PV FAULT DIAGNOSIS}

The selection of inputs directly bears on the effect of NNbased PV fault diagnosis. With appropriate inputs, the fault diagnosis model can obtain abundant information and make accurate diagnosis [15].

Firstly, short-circuit current $I_{s c}$ and open-circuit voltage $U_{o c}$ were taken as the inputs of PV fault diagnosis model. The former increases with illumination intensity, and the latter decrease with the growth in ambient temperature. Since the output power of PV power station is promoted by illumination intensity and suppressed by ambient temperature, the two selected inputs cover the information on the illumination intensity and ambient temperature.

Once PV modules fail, the output power of PV power station will not only decline, but also be affected by different types of faults to varied degrees. Figure 1 presents the voltagecurrent (U-I) curves for various types of PV faults, when the illumination intensity and ambient temperature remain constant.

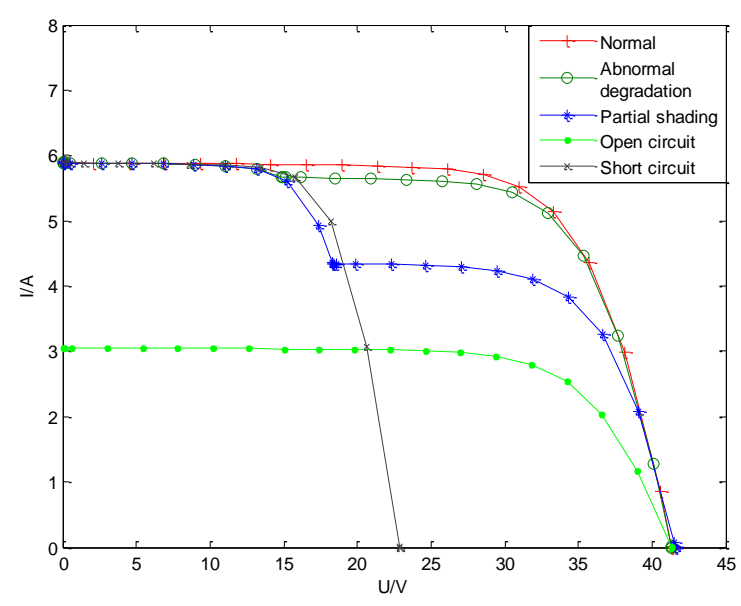

Figure 1. U-I curves under various types of faults

As shown in Figure 1, under the standard testing condition (STC: $1,000 \mathrm{~W} / \mathrm{m}^{2}, 2{ }^{\circ} \mathrm{C}$ ), the short-circuit current remained the same, while the open-circuit voltage dropped, in normal state and short-circuit state; the open-circuit voltage remained constant, whereas the short-circuit current declined, in normal and open-circuit state [16]. The variation of U-I curves for PV modules might be caused by fault, illumination intensity or ambient temperature, and cannot be used as the sole criterion to judge the fault type.

It can also be seen from Figure 1 that, the open-circuit voltage of the PV power station plunged at short-circuit fault; the short-circuit current decreased deeply at open-circuit fault; the maximum power voltage $U_{m p p}$ and maximum power current $I_{m p p}$ dropped significantly from the normal levels if a PV module suffered from abnormal degradation; the maximum power voltage $U_{m p p}$ remained at the short-circuit level, but the open-circuit voltage surpassed the short-circuit level, under the condition of partial shading.

In addition, one or more outputs changed significantly with fault conditions. Therefore, this paper selects four parameters as the inputs of PV fault diagnosis model, namely, maximum power voltage $U_{m p p}$, maximum power current $I_{m p p}$, shortcircuit current $I_{s c}$ and open-circuit voltage $U_{o c}$.
The NN is a popular and desirable tool to diagnose faults, especially those with complex nonlinearity. Considering the nonlinear property of PV faults, it is suitable to implement the $\mathrm{NN}$ to solve PV fault diagnosis.

\subsection{Defects of the BPNN}

The BPNN, known for its strength in nonlinear mapping, can effectively handle nonlinear problems [13]. However, there are several defects with the BPNN: slow and inaccurate convergence, and proneness to fall into the local optimum trap (that is, the gradient descent method in the BPNN ensures that network weights could converge to a solution, but not necessarily the global minimum point of the error hyperplane).

Many improved algorithms have been developed to overcome these defects, such as the additional momentum method, self-adaptive learning rate method, the resilient backpropagation (BP) method. But these improved algorithms still face long training time and low precision, if applied to diagnose PV faults.

In this paper, the PNN is employed to eliminate the defects of the BPNN, that is, to speed up the convergence and improve the training accuracy [17].

\subsection{Principle of the PNN}

The PNN is an NN specialized for pattern classification. Essentially a parallel algorithm, the PNN evolves from the classification rules of Bayesian function and probability density function (PDF) of Parzen window [18].

During network training, the PNN does not modify the vectors of the training sample, but saves them as the pattern sample. Then, the smoothing factor of Gaussian function will make estimation based on empirical statistics. Once the PNN starts working, the unknown sample $X$ will be sent directly from the input layer to each class on the pattern layer, and the vector dot product of $X$ and $W$ (weights) will be taken in the unit of pattern layer. After the nonlinear processing completes, the values of dot product will be put into the summation layer. In the summation layer, each unit is only connected with the corresponding unit in the pattern layer, and the probability of various types is estimated by the Parzen summation method. In the output layer (a.k.a. the decision-making layer), the input vectors were assigned to the class with the maximum posterior probability by Bayesian classification rules, according to their probability statistics.

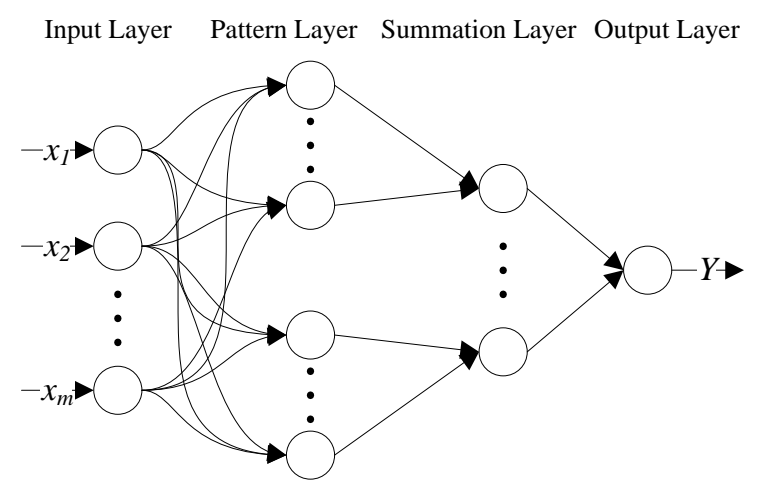

Figure 2. The structure of the PNN-based fault diagnosis model 
Unlike the BPNN, the PNN is a feedforward NN extended from the radial basis function (RBF), drawing on structural risk minimization, i.e. Bayesian decision theory. It inherits the advantages of the RBF network in pattern classification. If the distribution density Spread is close to zero, the PNN becomes a neighbor classifier; if the Spread is relatively large, the network serves as a near classifier. In our research, the PNNbased fault diagnosis model consists of such four layers as the input layer, the pattern layer, the summation layer and the output layer (Figure 2).

The first layer is the input layer, in which the number of neurons equals the dimension of samples. This layer mainly receives the data from the training samples and passes the eigenvector to the network.

The second layer is the pattern layer, in which the number of neurons equals the total number of samples in various classes. This layer computes the matching relationship between the input eigenvector and the sample of each fault mode. The output of each pattern layer neuron can be described as:

$$
f\left(X, W_{i}\right)=\exp \left[-\frac{\left(X-W_{i}\right)^{T}\left(X-W_{i}\right)}{2 \delta^{2}}\right]
$$

where $W_{i}$ is the weight of the edge between the input layer and pattern layer; $\delta$ is the smoothing factor that determines the width of the bell-mouthed curves at the center of all sampling points.

The third layer is the summation layer. As its name suggests, this layer adds up the probability of samples in the same fault mode, which is computed by (1), outputting the PDF of fault modes. Each class has only one neuron in the summation layer. Therefore, the number of neurons of this layer only depends on the number of pattern layer neurons belonging to their own type. In other words, the summation layer simply focuses on the output of pattern layer neurons of the same type. The output of summation layer is positively correlated to the PDF based on kernel functions. Then, the estimated probability for each type can be acquired by normalization in the output layer.

The fourth layer is the output layer, which consists of threshold identifiers. The function of this layer is to choose the neurons with maximum posterior probability density, and output them as the estimated probability density of various fault types. Each output neuron describes a competitive level related to the data type (fault mode). The number of output layer neurons equals the number of classes of the training samples. Together, the output layer neurons receive all types of PDFs outputted by the summation layer. The output neuron with the largest PDF is 1 and the corresponding type is the pattern class to be identified. Meanwhile, all the other output neurons output zeros.

The number of pattern layer neurons increases with that of fault samples. If there are more than two fault modes, the number of neurons in the summation layer will increase. As a result, with the accumulation of empirical knowledge, the PNN will expand horizontally and grow in the ability to diagnose faults.

The PNN-based fault diagnosis is a very popular decisionmaking method in probability statistics. Below is the mathematical description of this method. Given fault modes $\theta_{\mathrm{A}}$ and $\theta_{\mathrm{B}}$, the sample features of fault $X=\left(x_{1}, x_{2}, \ldots, x_{\mathrm{n}}\right)$ can be judged in two separate cases: if $h_{\mathrm{A}} l_{\mathrm{A}} f_{\mathrm{A}}(X)>h_{\mathrm{B}} l_{\mathrm{B}} f_{\mathrm{B}}(X)$, then $X$ $\in \theta_{\mathrm{A}}$; if $h_{\mathrm{A}} l_{\mathrm{A}} f_{\mathrm{A}}(X)<h_{\mathrm{B}} l_{\mathrm{B}} f_{\mathrm{B}}(X)$, then $X \in \theta_{\mathrm{B}}$. Note that $h_{\mathrm{A}}$ and $h_{\mathrm{B}}$ are the prior probabilities of fault modes $\theta_{\mathrm{A}}$ and $\theta_{\mathrm{B}}$, respectively $\left(h_{\mathrm{A}}=N_{\mathrm{A}} / N, h_{\mathrm{B}}=N_{\mathrm{B}} / N\right) ; N_{\mathrm{A}}$ and $N_{\mathrm{B}}$ are the training samples of fault modes $\theta_{\mathrm{A}}$ and $\theta_{\mathrm{B}}$, respectively; $N$ is the total number of samples; $l_{\mathrm{A}}$ is the cost of fault mode $\theta_{\mathrm{A}}$ and error of fault mode $\theta_{\mathrm{B}} ; l_{\mathrm{B}}$ is the cost of fault mode $\theta_{\mathrm{B}}$ and error of fault mode $\theta_{\mathrm{A}} ; f_{\mathrm{A}}$ and $f_{\mathrm{B}}$ are the PDFs of fault modes $\theta_{\mathrm{A}}$ and $\theta_{\mathrm{B}}$, respectively.

In general, the PDF can be estimated accurately, and its statistic can only be derived from the existing fault samples. The PDF estimation can be conducted by the Parzen method [19]:

$$
f_{A}(X)=\frac{1}{(2 \pi)^{P / 2} \delta^{P}} \frac{1}{m} \sum \exp \left[-\frac{\left(X-X_{a i}\right)^{T}\left(X-X_{a i}\right)}{2 \delta^{2}}\right]
$$

where $P$ is the dimension of vector $X$ and training vectors; $X_{\mathrm{ai}}$ is the $i$-th training vector of a fault mode; $m$ is the number of training samples of a fault mode; $\delta$ is the smoothing factor.

Normalizing all training vectors and undiagnosed vectors, we have:

$$
\left(X-X_{a i}\right)^{T}\left(X-X_{a i}\right)=-2\left(X^{T} X_{a i}-1\right)
$$

Considering that

$$
h_{A}=N_{A} / N
$$

Substituting (2) (4) to (1), the diagnosis rules of fault mode $a$ will change as follows. If $X$ belongs to $a$, then:

$$
L_{a} \sum_{i=1}^{N_{A}} \exp \left[\frac{X^{T} X_{a i}-1}{\delta^{2}}\right] \geq L_{b} \sum_{i=1}^{N_{B}} \exp \left[\frac{X^{T} X_{b i}-1}{\delta^{2}}\right]
$$

Then, the input layer receives and normalizes the input vector. Then, the response function of pattern layer can be expressed as:

$$
Y_{a i}=\exp \left[\frac{X^{T} X_{a i}-1}{\delta^{2}}\right]
$$

\subsection{Model description and data normalization}

Figure 3 shows the flow chart of the PNN-based diagnosis model.

As mentioned before, the inputs of the PNN-based diagnosis model include as $U_{m p p}, I_{m p p}, I_{s c}$ and $U_{o c}$ (Table 1) and the outputs are the fault states that can be distinguished by the model, namely, short circuit, open circuit, abnormal degradation and partial shading (Table 2).

The four inputs have different units and orders of magnitude. If inputted directly, the PNN will perform poorly in convergence. Therefore, before PNN training, the input data were normalized into the interval $[0,1]$ by:

$$
P_{n}=\frac{I_{n}-I_{\text {min }}}{I_{\text {max }}-I_{\text {min }}}
$$

where $I_{n}$ is the original input data; $I_{\max }$ and $I_{\min }$ are maximum and minimum of the input data, respectively; $P_{n}$ is the normalized input data. The same $I_{\max }$ and $I_{\min }$ were adopted for training and testing after the normalization. 
Table 1. Inputs of the PNN-based diagnosis model

Table 2. Outputs of the PNN-based diagnosis model

\begin{tabular}{cc}
\hline Input variable & Name of input variable \\
\hline$U_{\mathrm{mpp}}$ & Maximum power voltage \\
$I_{\mathrm{mpp}}$ & Maximum power current \\
$I_{\mathrm{sc}}$ & Short circuit current \\
$U_{\mathrm{oc}}$ & Open circuit voltage \\
\hline
\end{tabular}

\begin{tabular}{cccccc}
\hline Number & $\mathbf{O}_{1}$ & $\mathbf{O}_{2}$ & $\mathbf{O}_{3}$ & $\mathbf{O}_{4}$ & Fault mode \\
\hline 1 & 0 & 0 & 0 & 0 & Normal \\
2 & 1 & 0 & 0 & 0 & Short circuit \\
3 & 0 & 1 & 0 & 0 & Open circuit \\
4 & 0 & 0 & 1 & 0 & Abnormal degradation \\
5 & 0 & 0 & 0 & 1 & Partial shading \\
\hline
\end{tabular}

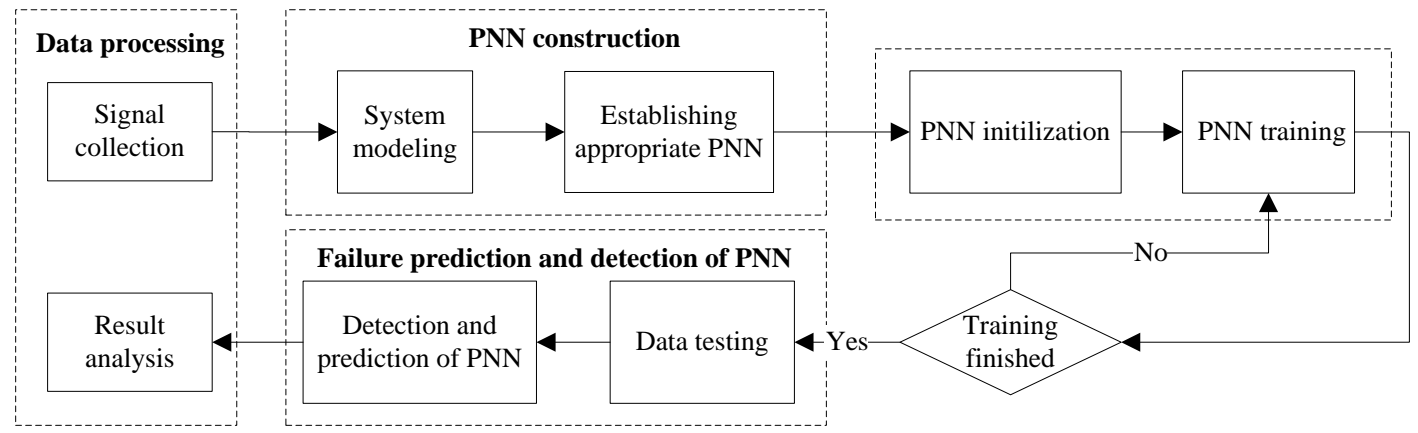

Figure 3. Flow chart of the PNN-based diagnosis model

\section{MODEL VERIFICATION}

To verify its effectiveness, our PNN-based fault diagnosis model was verified through both simulation and experiment. As shown in Figure 4, the PNN-based fault diagnosis model contains $3 * 3$ PV modules connected in series and parallel, a U-I curve collection module and a PNN module. The outputs were displayed to alert the responsible person to locate the detected faults.

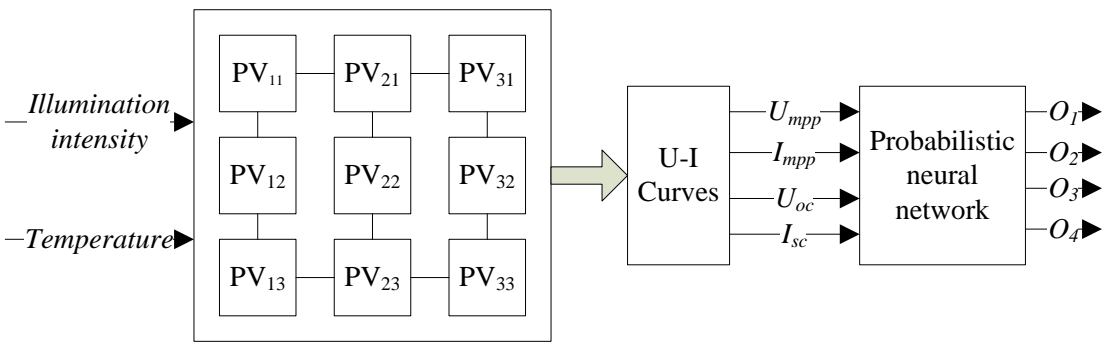

Figure 4. PNN-based fault diagnosis model

\subsection{Simulation verification}

Firstly, our model was verified through MATLAB/Simulink simulation. The PNN training data include the input data of $U_{m p p}, I_{m p p}, I_{s c}$ and $U_{o c}$ under the illumination intensity of $200 \sim 1,000 \mathrm{~W} / \mathrm{m}^{2}$ and the ambient temperature of $25 \sim 45{ }^{\circ} \mathrm{C}$. The U-I curve collection module gathered 450 datasets. Among them, 400 datasets were taken as training samples and the others as testing samples. The Spread was set empirically to 0.8 . The Gaussian function and Euclidean distance were respectively taken as the activation function and the weighting function, respectively. In addition, netprod and compet were selected as input and output functions, respectively.

The results and errors of PNN training are displayed in Figures 5 and 6 , respectively. Note that the red curves are actual faults, while the blue ones are the diagnosis results. After PNN training, the trained model was imported with the testing data.

Based on 50 testing datasets, the trained PNN was applied to detect the fault types of PV modules. Figure 7 shows some of the diagnosis results. Note that the red curves are actual faults, while the blue ones are the diagnosis results. As shown in Figure 7, the PNN outputs were consistent with the predefined outputs under each type of fault, and the diagnosis accuracy reached $60.0 \%$, indicating that the PNN is feasible for PV fault diagnosis.

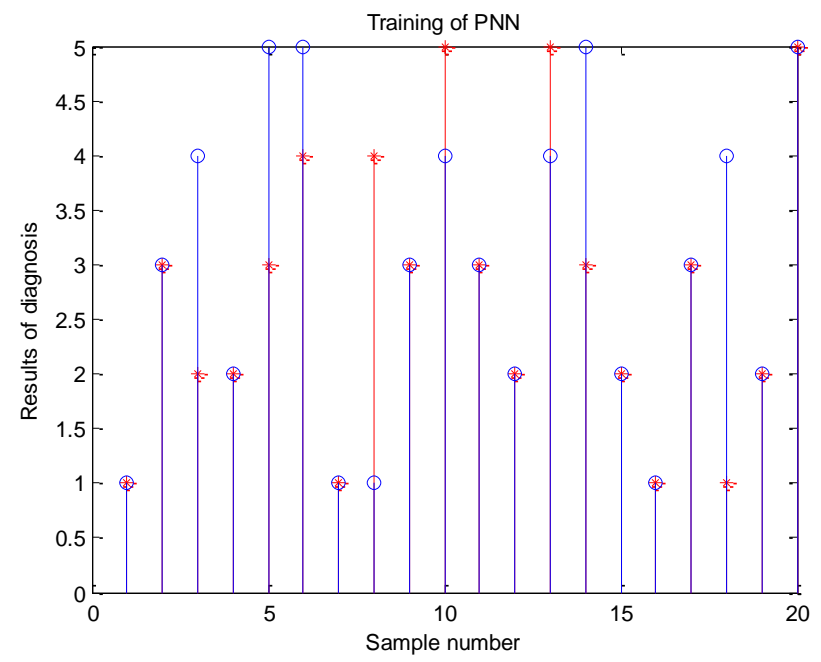

Figure 5. Diagnosis results of PNN training 


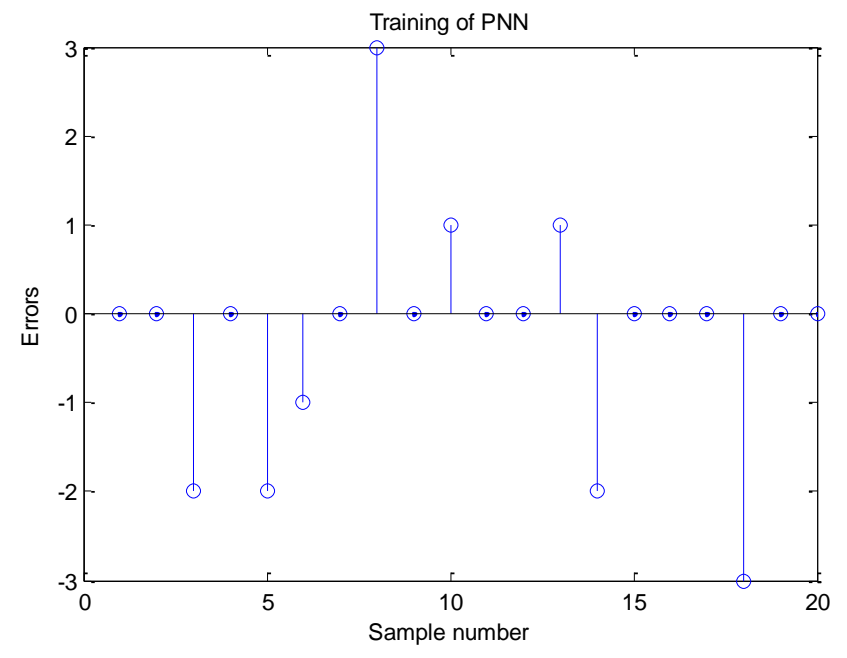

Figure 6. Errors of PNN training

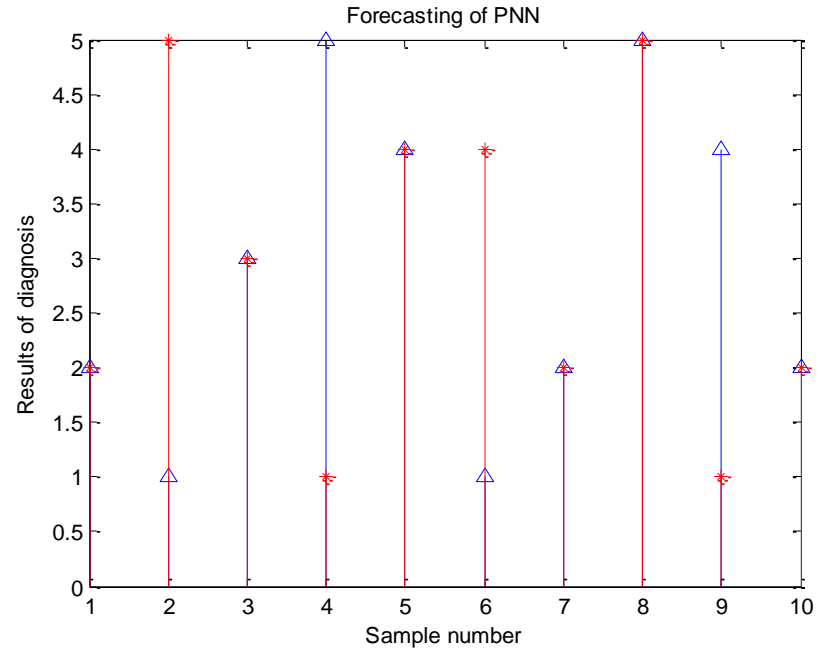

Figure 7. PNN-based diagnosis results

Table 3. Fault test samples of PV modules

\begin{tabular}{ccccccccc}
\hline Number & $\boldsymbol{I}_{\boldsymbol{s c}} / \mathbf{A}$ & $\boldsymbol{I}_{\boldsymbol{m p p}} / \mathbf{A}$ & $\boldsymbol{U}_{\boldsymbol{o c}} / \mathbf{V}$ & $\boldsymbol{U}_{\boldsymbol{m p p}} / \mathbf{V}$ & $\begin{array}{c}\text { Short } \\
\text { circuit }\end{array}$ & $\begin{array}{c}\text { Open } \\
\text { circuit }\end{array}$ & $\begin{array}{c}\text { Abnormal } \\
\text { degradation }\end{array}$ & $\begin{array}{c}\text { Partial } \\
\text { shading }\end{array}$ \\
\hline 1 & 5.928 & 5.631 & 41.53 & 31.26 & 0 & 0 & 0 & 0 \\
2 & 3.140 & 2.537 & 27.51 & 20.30 & 1 & 0 & 0 & 0 \\
3 & 4.520 & 3.744 & 40.63 & 26.91 & 0 & 1 & 0 & 0 \\
4 & 1.917 & 1.581 & 40.33 & 29.79 & 0 & 0 & 1 & 0 \\
5 & 5.218 & 4.762 & 41.21 & 29.81 & 0 & 0 & 0 & 1 \\
\hline
\end{tabular}

Five typical fault test samples (Table 3) were selected for PNN-based fault diagnosis. The diagnosis results are presented in Table 4 below.

Table 4. PNN-based diagnosis results

\begin{tabular}{ccccc}
\hline Number & $\begin{array}{c}\text { Short } \\
\text { circuit }\end{array}$ & $\begin{array}{c}\text { Open } \\
\text { circuit }\end{array}$ & $\begin{array}{c}\text { Abnormal } \\
\text { degradation }\end{array}$ & $\begin{array}{c}\text { Partial } \\
\text { shading }\end{array}$ \\
\hline 1 & 0 & 0 & 0 & 0 \\
2 & 1 & 0 & 0 & 0 \\
3 & 0 & 1 & 0 & 0 \\
4 & 0 & 0 & 1 & 0 \\
5 & 0 & 0 & 0 & 1 \\
\hline
\end{tabular}

According to the diagnosis results, the short circuit current $I_{s c}$ under the state of open circuit and the maximum power current $I_{m p p}$ differed greatly from those under the other states. Similarly, the open circuit voltage $U_{o c}$ under the state of short circuit and the maximum power voltage $U_{m p p}$ had marked differences from those under other states. Thus, the open circuit fault and short circuit fault could be classified easily by the trained model. Nonetheless, our model could not distinguish between other fault types easily, due to the complexity and the variability of partial shading and abnormal degradation [20].

(1) Analysis of abnormal degradation

With the elapse of time, the PV modules gradually age and output an increasingly low power. This aging phenomenon is known as abnormal degradation. For example, the PV modules may be eroded by water vapor and thus have a lower output power [21]. The maximum power voltage $U_{m p p}$ is the most vulnerable parameter to abnormal degradation.

The abnormal degradation is generally simulated by increasing the series resistance $R_{S}$ or decreasing the parallel resistance $R_{s h}$ [22]. The former strategy is relative more common. To determine the appropriate series resistance of abnormal degradation, the PV modules were simulated under the illumination intensity of $1,000 \mathrm{~W} / \mathrm{m}^{2}$. The resulting curves of $U_{m p p}$ at different series resistances are given in Figure 8.

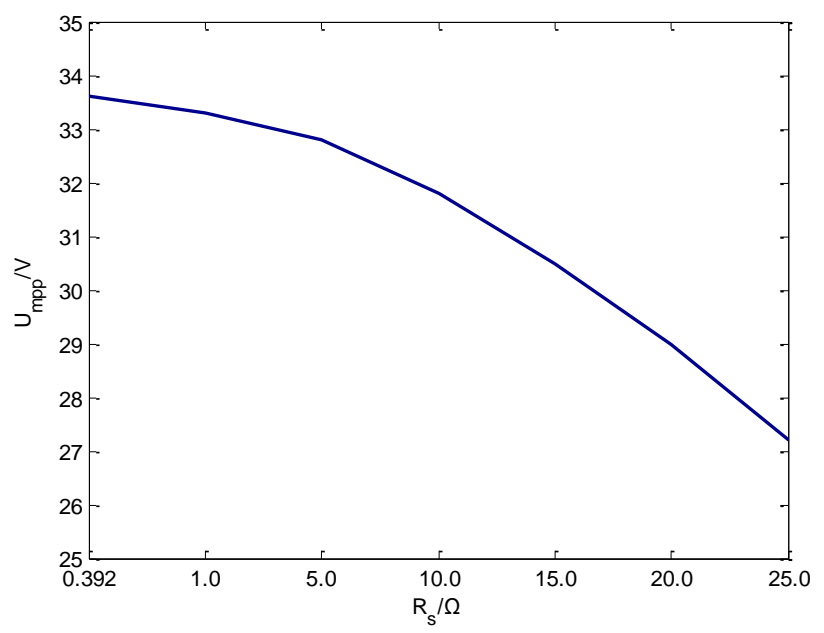

Figure 8. The $U_{m p p}$ at different series resistances

As shown in Figure 8, the maximum power voltage $U_{m p p}$ plunged deeply with the increase in series resistance, which favors the PNN classification. It can also be found that the $U_{m p p}$ value minimized at the series resistance of $25 \Omega$.

Figure 9 compares the $U_{m p p}$ under normal state and that under abnormal degradation at different illumination intensities. It can be seen that the $U_{m p p}$ in abnormal degradation was almost the same with that in normal condition under a low illumination intensity. That is why PV faults may be misdiagnosed at a low illumination intensity. However, the fault of abnormal degradation can be identified easily when the illumination intensity is high.

(2) Analysis of partial shading 
In real-world cases, partial shading often occurs due to the shadow of various objects (e.g. trees, buildings and clouds), manufacturing defects, cracking, etc. [23] The wide presence of these objects complicate the analysis of partial shading in PV fault diagnosis. Under partial shading, the PV modules may have different output features, and the output power of the entire PV power station may be limited to the PV module with the smallest output power. In addition, partial shading will cause a huge variation in the maximum output power of PV modules.

To describe partial shading, the parameter $\alpha$ was introduced as the rate of shadow blocked without considering temperature variation. If $\alpha=0.1$, then $10 \%$ of irradiance is blocked and the transmission of irradiance is $(1-\alpha)$. Previous simulations have shown that the PNN detected the fault types correctly when $\alpha$ varied from 0.4 to 0.7 , but failed to do so under the other conditions. Here, the value of $\alpha$ is set to 0.5 . In this case, the misdiagnosis is mainly the result of the lack of training samples. To enhance the PNN performance, it is necessary to collect more output data under different $\alpha$ values for PV modules.

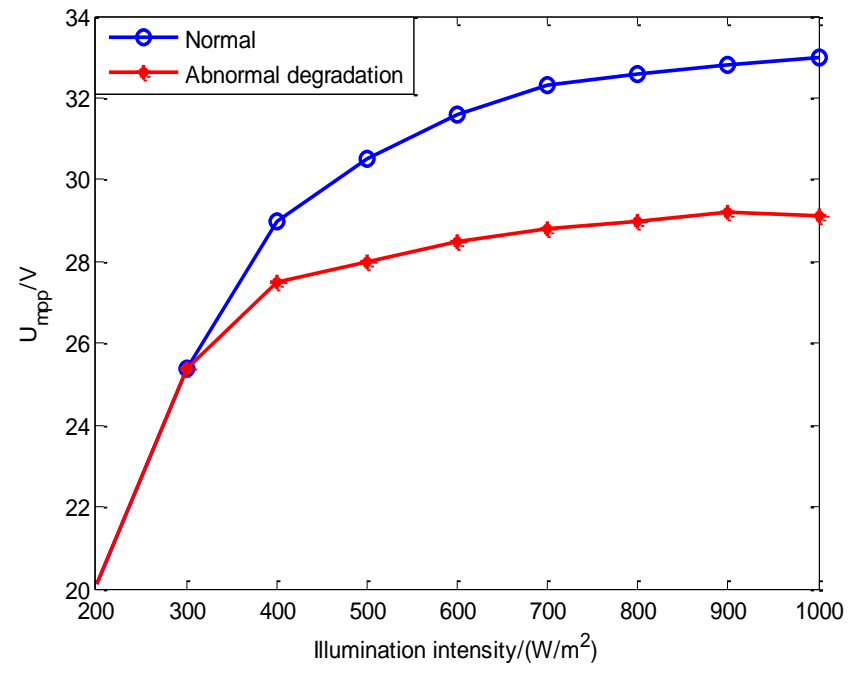

Figure 9. The $U_{m p p}$ at different illumination intensities

\subsection{Experimental verification}

Our model was further validated on an experimental platform, consisting of a data acquisition module and a fault diagnosis module (Figure 10) [24]. The test object is a group of $3 * 3 \mathrm{PV}$ modules with the same parameters and connected in series and parallel.

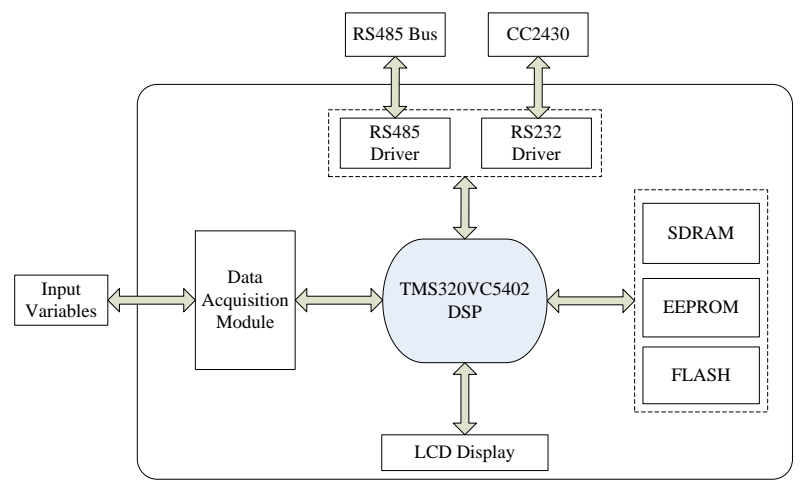

Figure 10. Block diagram of the experimental platform
The data from the fault diagnosis module were transmitted wireless to the host. The transmission mode is described in Figure 11 below.

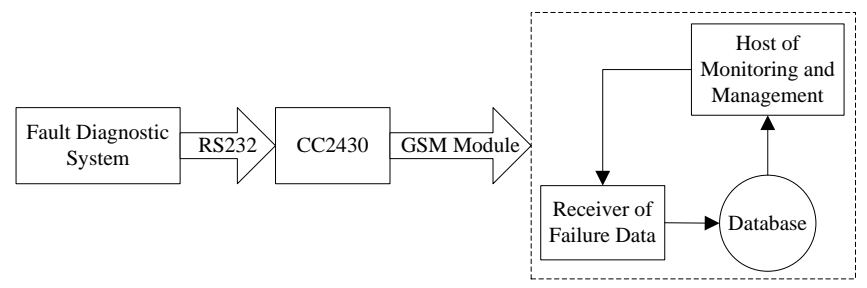

Figure 11. The wireless data transmission mode

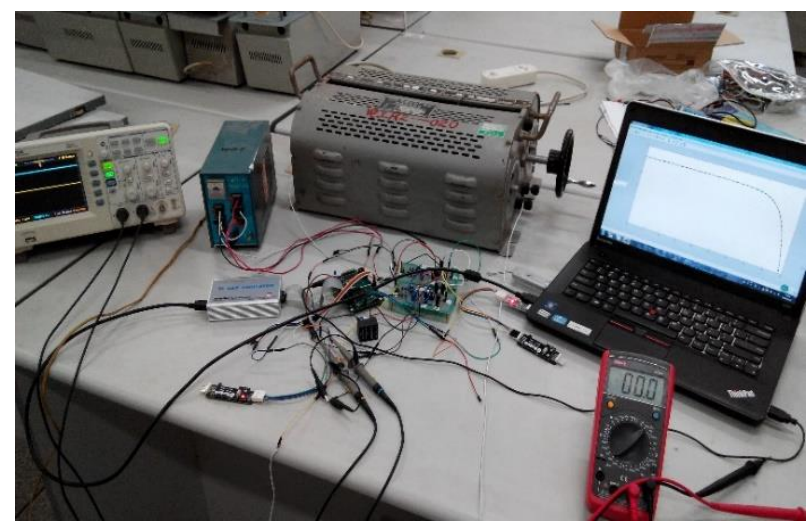

Figure 12. A photo of the experimental platform

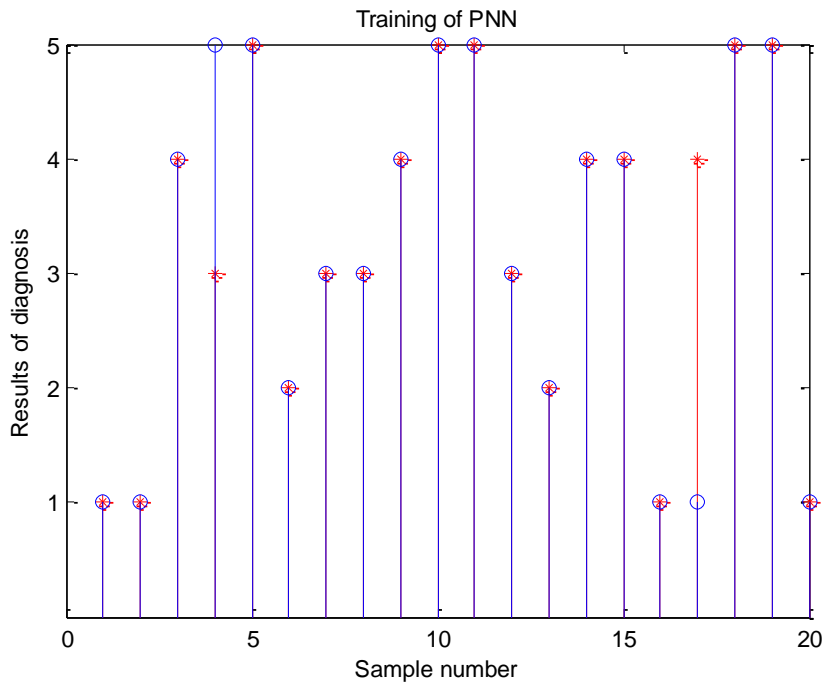

Figure 13. Diagnosis results of PNN training

Figure 12 is a photo of the experimental platform. To test the effectiveness of our model in different environments, the operating data in sunny and cloudy days were both collected by the data acquisition module. Meanwhile, the parameter values of the PV modules were gathered at various illumination intensities and ambient temperatures [25]. A total of 500 datasets were obtained after repeated measurements. Among them, 400 datasets were taken as the training sample, and the remaining 100 as the testing sample. Five typical fault test samples (Table 5) were selected for the PV fault diagnosis by our model. The fault test samples, diagnosis results and errors of PNN training were displayed in Figures 13 and 14, respectively, and the $\mathrm{PNN}$-based diagnosis results are shown in Figure 15. 
Table 5. Fault test samples of PV modules

\begin{tabular}{ccccccccc}
\hline Number & $\boldsymbol{I}_{\boldsymbol{s c}} / \mathbf{A}$ & $\boldsymbol{I}_{\boldsymbol{m} \boldsymbol{p p}} / \mathbf{A}$ & $\boldsymbol{U}_{\boldsymbol{o c}} / \mathbf{V}$ & $\boldsymbol{U}_{\boldsymbol{m p p}} / \mathbf{V}$ & $\begin{array}{c}\text { Short } \\
\text { circuit }\end{array}$ & $\begin{array}{c}\text { Open } \\
\text { circuit }\end{array}$ & $\begin{array}{c}\text { Abnormal } \\
\text { degradation }\end{array}$ & $\begin{array}{c}\text { Partial } \\
\text { shading }\end{array}$ \\
\hline 1 & 5.305 & 4.938 & 35.43 & 29.33 & 0 & 0 & 0 & 0 \\
2 & 4.831 & 2.963 & 30.21 & 25.46 & 1 & 0 & 0 & 0 \\
3 & 3.658 & 3.204 & 32.86 & 26.81 & 0 & 1 & 0 & 0 \\
4 & 3.510 & 2.979 & 33.92 & 24.85 & 0 & 0 & 1 & 0 \\
5 & 4.659 & 3.195 & 35.05 & 24.78 & 0 & 0 & 0 & 1 \\
\hline
\end{tabular}

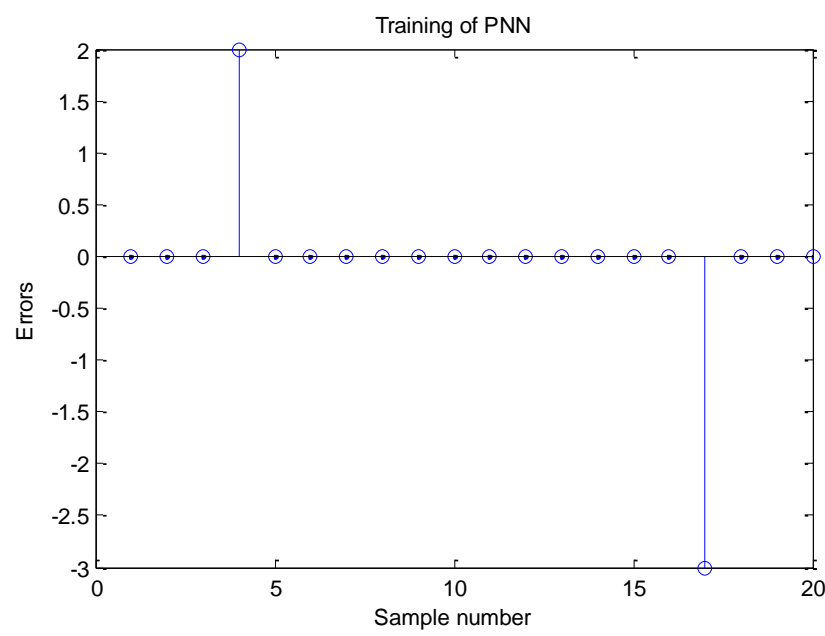

Figure 14. Errors of PNN training

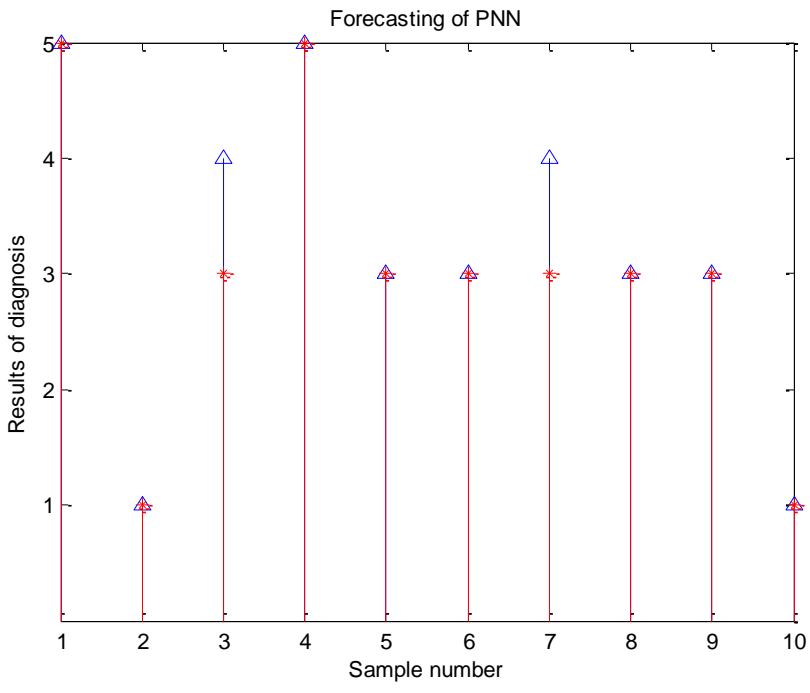

Figure 15. PNN-based diagnosis results
The results show that our model achieved an up to $90.0 \%$ diagnosis accuracy, revealing the importance of parameters like $R_{s}$ and $\alpha$ in PV fault diagnosis.

Figure 16 shows the precision of fault diagnosis of our model at different fault states. It can be seen that our model successfully detected fault types like short circuit and open circuit. Some misdiagnoses still occurred in the states of normal, abnormal degradation and partial shading, owing to the complexity of the external environment. Overall, the experimental results are relatively satisfactory.

The diagnosis accuracy can be greatly affected by Spread, the expansion coefficient of RBF. The default value of Spread is 1.0. If it is too small, the RBF neurons will not respond to the interval covered with the input vectors; If it is too large, the computing load will be too heavy. In generally, the greater the Spread, the smoother the outputs. The optimal Spread is less than the typical distance among the input vectors. The influence of Spread over diagnosis accuracy is described in Table 6 .

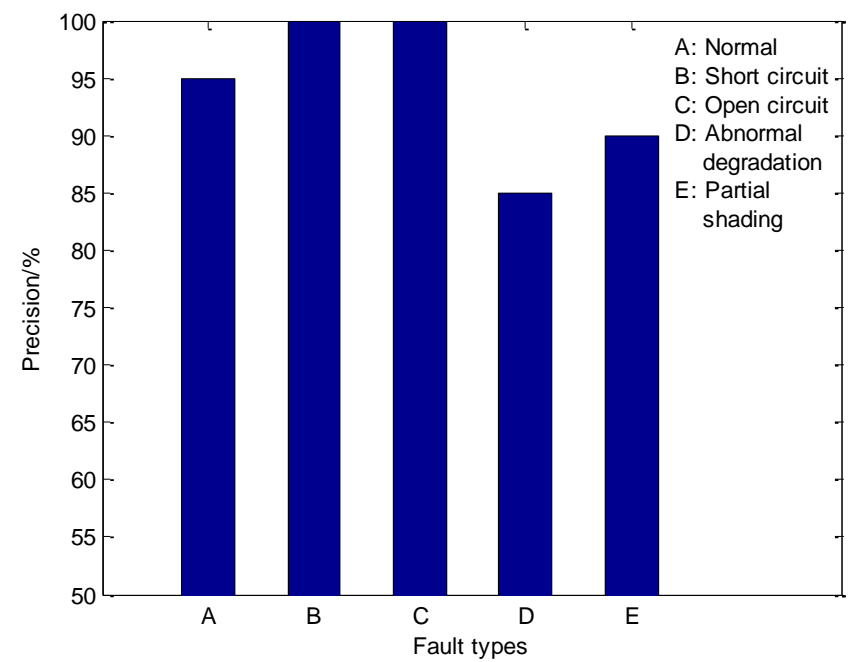

Figure 16. Precision of fault diagnosis

Table 6. The influence of Spread over diagnosis accuracy

\begin{tabular}{ccccccc}
\hline \multirow{2}{*}{ Spread } & \multicolumn{5}{c}{ Diagnosis accuracies/\% } \\
\cline { 2 - 7 } & Normal & $\begin{array}{c}\text { Short } \\
\text { circuit }\end{array}$ & $\begin{array}{c}\text { Open } \\
\text { circuit }\end{array}$ & $\begin{array}{c}\text { Abnormal } \\
\text { degradation }\end{array}$ & $\begin{array}{c}\text { Partial } \\
\text { shading }\end{array}$ & $\begin{array}{c}\text { Total } \\
\text { accuracy }\end{array}$ \\
\hline 0.01 & 70.0 & 100 & 95.0 & 50.0 & 70.0 & 77.0 \\
0.1 & 70.0 & 100 & 100 & 55.0 & 70.0 & 79.0 \\
0.4 & 85.0 & 100 & 100 & 75.0 & 80.0 & 88.0 \\
0.8 & 95.0 & 100 & 100 & 85.0 & 90.0 & 94.0 \\
1.0 & 95.0 & 100 & 100 & 85.0 & 85.0 & 93.0 \\
\hline
\end{tabular}

\subsection{Comparison between BPNN and PNN}

As shown in Table 7, the PNN has the following advantages than the BPNN.

(1) The PNN has a simpler and faster convergence process than the BPNN. Despite having the same input and output, the BPNN and the PNN differ in many aspects. For instance, the number of hidden layers in the BPNN is empirical and selected non-deterministically through trial-and-error, while that in the PNN is basically fixed. Besides, the PNN does not need to 
determine the number of hidden layer neurons. Furthermore, the BPNN's learning algorithm converges slowly and often falls into the local optimum trap. By contrast, the PNN training avoids the local optimum trap and completes very quickly, because the training is a one-step process and the samples are directly assigned.

Table 7. Experimental results of BPNN and PNN

\begin{tabular}{ccc}
\hline \multirow{2}{*}{ Algorithm } & \multicolumn{2}{c}{ Parameter } \\
\cline { 2 - 3 } & Convergence time/s & Precision/\% \\
\hline BP & 1.72 & 66.7 \\
PNN & 0.75 & 85.0 \\
\hline
\end{tabular}

(2) The PNN consistently converges to the optimal solution of Bayesian rules. The BPNN classification rules are too ambiguous to output transparent results, while the PNN classifies the objects by the risk minimization rules of Bayesian and uses the prior knowledge. However complex the classification problem, the PNN can always converge to the optimal solution of Bayesian rules, provided that there are sufficient training samples. On the contrary, the BPNN may stop at a local optimal solution rather than converge to the global optimum.

(3) The PNN works well with additional samples and even tolerates some wrong samples. This network only needs to increase or decrease the number of neurons in the pattern layer, in order to add new samples or remove old samples. The new samples can be added by assigning new connection weights between input and pattern layers. For the BPNN, however, the network must be trained again whenever the training samples are modified, and the connection weights must be reassigned. This is basically rebuilding the entire network.

(4) The PNN can process abnormal data in real time. If it is applied to classify abnormal data, what is originally completed by nonlinear learning algorithm can be done by linear learning algorithm, such as to maintain the high diagnosis accuracy. Moreover, the PNN does not need to be trained again.

In actual PV fault diagnosis, the fault samples of PV modules are constantly changing, which gives full play to the superiority of the PNN in handling additional samples. To sum up, the PNN-based model outperforms the BPNN in multiple aspects of fault diagnosis, including but not limited to fast diagnosis, high accuracy and the tolerance of new samples.

\section{CONCLUSIONS}

The PNN-based fault diagnosis makes full use of prior knowledge of faults and detects different types of faults deterministically under risk minimization of Bayesian rules. In this paper, a PV fault diagnosis model is proposed to detect four types of faults, namely, open circuit, short circuit, abnormal degradation and partial shading. Both simulation and experiment show that our model detected different types of PV modules effectively, enabling the responsible person to locate the faults and take countermeasures in time. The results analysis show that the PNN enjoys high training speed and is easy to implement in engineering. Moreover, the PNN-based fault diagnosis has strong robustness and high accuracy. With the accumulation of fault knowledge, our model requires no additional instruments, and supports the online detection. Compared with the BPNN-based diagnosis model, our model is obvious superior in convergence speed and precision. Future research will further improve our model to tackle the uncertainty and complexity of PV faults induced by environmental factors.

\section{ACKNOWLEDGEMENTS}

This work is supported by Natural Science Research Program of Huai'an City (No. HAB201831). The authors would like to thank the editor and anonymous reviewers for their suggestions in improving the quality of the paper.

\section{REFERENCES}

[1] Pappas, C., Karakosta, C., Marinakis, V., Psarras, J. (2012). A comparison of electricity production technologies in terms of sustainable development. Energy Conversion and Management, 64: 626-632. https://doi.org/10.1016/j.enconman.2012.06.006

[2] Razykov, T.M., Ferekides, C.S., Morel, D., Stefanakos, E. (2011). Solar photovoltaic electricity: Current status and future prospects. Solar Energy, 85(8): 1580-1608. https://doi.org/10.1016/j.solener.2010.12.002

[3] Gokmen, N., Karatepe, E., Silvestre, S., Celik, B., Ortega P. (2013). An efficient fault diagnosis method for PV systems based on operating voltage-window. Energy Conversion and Management, 73: 350-360. https://doi.org/10.1016/j.enconman.2013.05.015

[4] Wang, P.Z., Zheng, S.C. (2010). Fault analysis of photovoltaic array based on infrared image. Acta Energiae Solaris Sinica, 31(2): 197-201.

[5] Chao, K., Ho, S., Wang, M. (2008). Modeling and fault diagnosis of a photovoltaic system. Electric Power Systems $\quad$ Research, 78(1): 97-105. https://doi.org/10.1016/j.epsr.2006.12.012

[6] Chouder, A., Sliverstre, S. (2010). Automatic supervision and fault detection of PV systems based on power losses analysis. Energy Conversion and Management, $\quad$ 51(10): https://doi.org/10.1016/j.enconman.2010.02.025

[7] Schirone, L., Califano, F.P. (1994). Fault finding in a 1 MW photovoltaic plant by reflectometry. IEEE Photovoltaic Energy Conversion, 1(1): 846-849. https://doi.org/10.1109/WCPEC.1994.520093

[8] Takumi, T., Junji, Y., Masayoshi, I. (2008). Disconnection detection using earth capacitance measurement in photovoltaic module string. Progress in Photovoltaic, 16(8):

https://doi.org/10.1002/pip.860

669-677.

[9] Ben, S.C., Ouali, M. (2011). Comparison of fuzzy logic and neural network in maximum power point tracker for PV systems. Electric Power Systems Research, 81(1): 43-50. https://doi.org/10.1016/j.epsr.2010.07.005

[10] Chao, K.H., Liao, B.J., Hung, C.P. (2013). Applying a cerebellar model articulation controller neural network to a photovoltaic power generation system fault diagnosis. International Journal of Photoenergy, 2013. http://doi.org/10.1155/2013/839621

[11] Firth, S.K., Lomas, K.J., Rees, S.J. (2010). A simple model of PV system performance and its use in fault detection. Solar Energy, 84(4): 624-635. https://doi.org/10.1016/j.solener.2009.08.004 
[12] Wang, M.H., Chen, M.J. (2012). Two-stage fault diagnosis method based on the extension theory for PV power systems. International Journal of Photoenergy, 2012. http://doi.org/10.1155/2012/892690

[13] Shan, F.H., Zhao, L.Q., Yang, F. (2018). A novel semantic matching method for chatbots based on convolutional neural network and attention mechanism. Revue d'Intelligence Artificielle, 32(S1): 103-114. https://doi.org/ 10.3166/RIA.32.S1.103-114

[14] Mostefa, T., Tarak, B., Hachemi, G. (2018). An automatic diagnosis method for an open switch fault in unified power quality conditioner based on artificial neural network. Traitement du Signal, 35(1): 7-21. https://doi.org/ 10.3166/TS.35.7-21

[15] Gokmen, N., Karatepe, E., Celik, B., Silvestre, S. (2012). Simple diagnostic approach for determining of faulted PV modules in string based on PV arrays. Solar Energy, 86(11): https://doi.org/10.1016/j.solener.2012.09.007

[16] Patel, H., Agarwal, V. (2008). MATLAB-based modeling to study the effects of partial shading on PV array characteristics. IEEE Transactions on Energy Conversion, 23(1): 302-310 https://doi.org/10.1109/TEC.2007.914308

[17] Specht, D.F., Shapiro, P.D. (1991). Generalization accuracy of probabilistic neural networks compared with back propagation networks. International Joint Conference on Neural Network (IJCNN), pp. 887-892. https://doi.org/10.1109/IJCNN.1991.155296

[18] Specht, D.F. (1990). Probabilistic neural networks and the polynomial Adaline as complementary techniques for classification. IEEE Transactions on Neural Networks, 1(1): 111-121. https://doi.org/10.1109/72.80210
[19] Parzen, E. (1962). On estimation of a probability density function and mode. Annals of Mathematical Statistics, 33(3): 1065-1076.

[20] Karatepe, E., Hiyama, T., Boztepe, M., Colak, M. (2008). Voltage based power compensation system for photovoltaic generation system under partially shaded isolation conditions. Energy Conversation and Management, $\quad$ 49(8): $\quad$ 2307-2316. https://doi.org/10.1016/j.enconman.2008.01.012

[21] Meyer, E.L., Vandyke, E.E. (2004). Assessing the reliability and degradation of photovoltaic module performance parameters. IEEE Transactions on Reliability, 53(1): 83-93. https://doi.org/10.1109/TR.2004.824831

[22] Scofield, J.H. (1995). Effects of series resistance and inductance on solar cell admittance measurements. Solar Energy Materials \& Solar Cells, 37: 217-233. https://doi.org/10.1016/0927-0248(95)00016-X

[23] Gao, L., Dougal, R.A., Liu, S., Iotova, A.P. (2009). Parallel-connected solar PV system to address partial and rapidly fluctuating shadow conditions. IEEE Transactions on Industrial Electronics, 56(5): 1548-1556. https://doi.org/10.1109/TIE.2008.2011296

[24] Vergura, S., Acciani, G., Amoruso, V., Patrono, G.E., Vacca, F. (2009). Descriptive and inferential statistics for supervising and monitoring the operation of PV plants. IEEE Transactions on Industrial Electronics, 56(11): 4456-4464. https://doi.org/10.1109/TIE.2008.927404

[25] Frank, P.M. (1994). On-line fault detection in uncertain nonlinear system using diagnostic observers: A survey. International Journal of Systems Science, 25(12): 21292154. https://doi.org/10.1080/00207729408949341 